TAMARA A. SUSSMAN, MD

Department of Internal Medicine,

Cleveland Clinic
NABIN K. SHRESTHA, MD

Department of Infectious Disease, Department of Clinical Pathology, Transplantation Center, and International Travel Clinic, Cleveland

Clinic; Associate Professor, Cleveland Clinic Lerner College of Medicine of Case Western

Reserve University, Cleveland, $\mathrm{OH}$
MARIA MIKLOWSKI, MD

Department of Internal Medicine, Cleveland Clinic; Clinical Assistant Professor, Cleveland Clinic Lerner College of Medicine of Case

Western Reserve University, Cleveland, $\mathrm{OH}$

\title{
Eyes of the mimicker
}

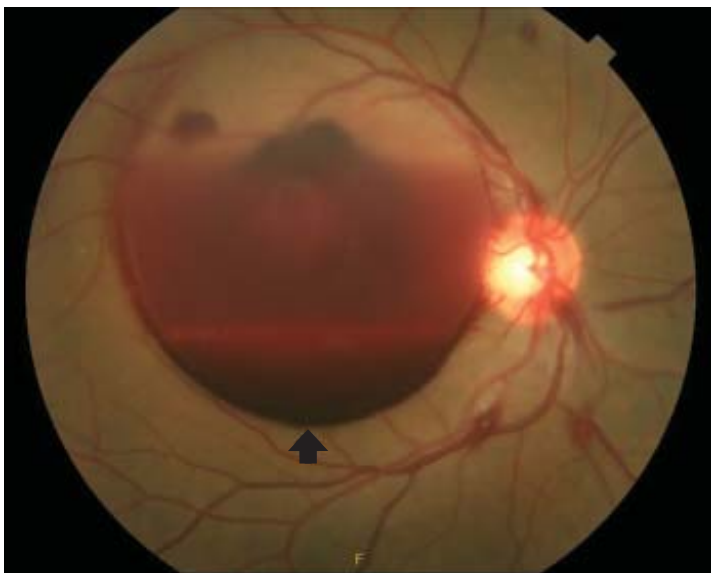

Figure 1. A dilated fundus photograph of the patient's right eye shows large subhyaloid hemorrhage (arrow).

\section{The CDC recommends annual syphilis testing for men who have sex with men}

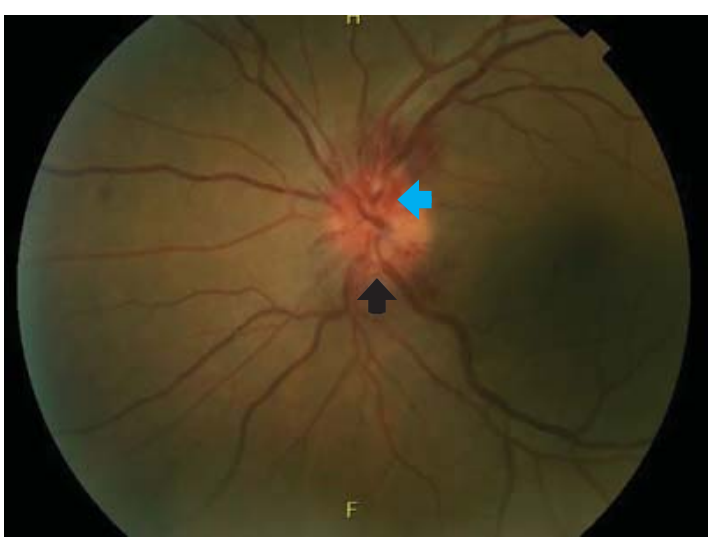

Figure 3. A dilated fundus photograph of the left eye shows optic disc edema (black arrow) with associated optic disc hemorrhages (blue arrow).

42-YEAR-OLD MAN presented with intermit-
tent fevers, rigors, headache, and myal-
gia for 6 days, followed by blurred vision in his
right eye with central vision loss for the past
several hours. He reported no associated pain,
flashes of light, or floaters. He was sexually ac-
tive with 1 male partner for 23 years. He had
doi:10.3949/ccjm.85a.17074

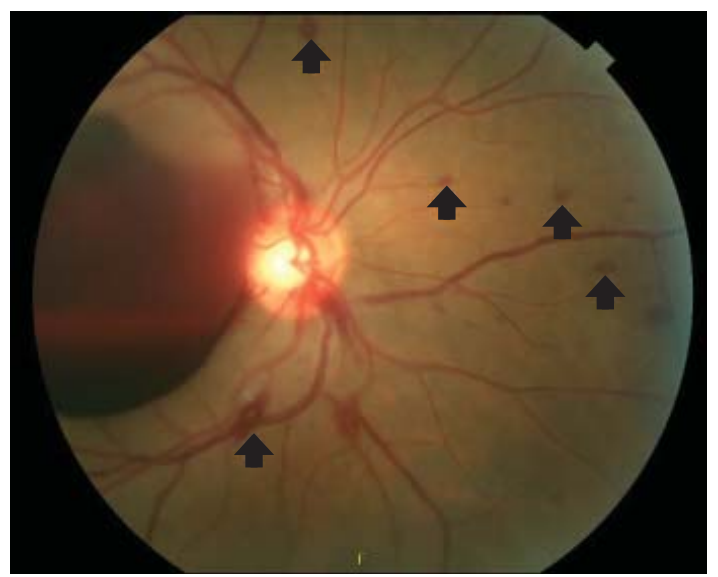

Figure 2. Multiple white-centered intraretinal hemorrhages, or Roth spots (arrows) were noted in the right eye.

no history of human immunodeficiency virus (HIV), sexually transmitted infections, or intravenous drug use.

Dilated funduscopy showed a large subhyaloid hemorrhage and retinal hemorrhages with white centers (Roth spots) in the right eye, and disc edema in the left eye (Figures 1-3).

On laboratory testing, serum rapid plasma reagin was reactive, Treponema pallidum immunoglobulin $\mathrm{G}$ was reactive (antibody index $>8.0$ ), and HIV testing was negative.

Lumbar puncture study revealed 34 nucleated cells/ $\mu \mathrm{L}$ (94\% lymphocytes), protein 58 $\mathrm{mg} / \mathrm{dL}$, and glucose $62 \mathrm{mg} / \mathrm{dL}$. Cerebrospinal fluid Venereal Disease Research Laboratory and fluorescent treponemal antibody absorption tests were reactive, confirming a diagnosis of ocular syphilis.

The patient was admitted to the hospital for treatment with intravenous penicillin G. After 5 days, he was discharged with instructions to complete a 10-day course of intravenous ceftriaxone (chosen for its ease of administration), for a total of 14 days of antibiotic therapy. His vision 
improved with treatment.

He continued to follow up with ophthalmology and infectious disease. Subsequent dilated fundus examinations showed resolution of pathology in the left eye, resolution of Roth spots in the right eye, and resolution of the subhyaloid hemorrhage. Repeat cerebrospinal fluid study examination was planned if the serum rapid plasma reagin had not become nonreactive 24 months after treatment.

\section{RECOGNIZING AND MANAGING OCULAR SYPHILIS AND NEUROSYPHILIS}

In addition to ocular syphilis and neurosyphilis, the differential diagnosis for Roth spots and disc edema on dilated funduscopy includes endocarditis, viral retinitis, and autoimmune or inflammatory conditions such as sarcoidosis and vasculitis.

In our patient, infectious endocarditis was considered, given his history of intermittent fevers and rigors, but it was ultimately ruled out by negative blood cultures and the absence of valvular vegetations on echocardiography.

The large subhyaloid hemorrhage raised suspicion of leukemia, but this was ruled out by the normal total white blood cell count and differential. HIV, herpetic retinitis, and toxoplasmosis were also considered, but labo-

\section{REFERENCES}

1. Fu EX, Geraets RL, Dodds EM, et al. Superficial retinal precipitates in patients with syphilitic retinitis. Retina 2010; 30(7):1135-1143. doi:10.1097/IAE.0b013e3181cdf3ae

2. US Centers for Disease Control and Prevention. Sexually Transmitted Diseases. Clinical Advisory: Ocular Syphilis in the United States, March 24, 2016. www.cdc.gov/std/syphilis/clinicaladvisoryos 2015 . htm. Accessed March 28, 2018.

3. US Centers for Disease Control and Prevention. Sexually Transmitted Disease Surveillance, 2015. www.cdc.gov/std/stats15/std-surveillance- ratory tests for these infections were negative.

Typically, retinal precipitates are more characteristic of syphilitic retinitis and distinguish it from other infectious causes such as herpetic retinitis and toxoplasmosis. ${ }^{1}$ Additionally, ocular syphilis more commonly manifests as uveitis or panuveitis. ${ }^{1,2}$ Our patient's ocular syphilis presented with white-centered retinal hemorrhages, subhyaloid hemorrhage, and optic disc edema.

\section{Who is at highest risk?}

About $90 \%$ of syphilis cases occur in men, and $81 \%$ occur in men who have sex with men. The US Centers for Disease Control and Prevention (CDC) thus recommends annual syphilis testing for men who have sex with men. ${ }^{3}$

Classically, syphilis was called "the great imitator" because it mimicked manifestations of other diseases. Patients with ocular manifestations of syphilis may not have other neurologic symptoms. ${ }^{4,5}$ Nevertheless, cerebrospinal fluid examination should be done in all instances of ocular syphilis, as many patients with ocular syphilis have evidence of neurosyphilis on testing. ${ }^{2}$ The $\mathrm{CDC}$ also recommends follow-up cerebrospinal fluid analysis to assess treatment response. ${ }^{2}$ This was planned in our patient.

2015-print.pdf. Accessed March 28, 2018.

4. Rishi E, Govindarajan MV, Biswas J, Agarwal M, Sudharshan S, Rishi P. Syphilitic uveitis as the presenting feature of HIV. Indian J Ophthalmol 2016; 64(2):149-150. doi:10.4103/0301-4738.179714

5. Zhang R, Qian J, Guo J, et al. Clinical manifestations and treatment outcomes of syphilitic uveitis in a Chinese population. J Ophthalmol 2016; 2016:2797028. doi:10.1155/2016/2797028

ADDRESS: Tamara A. Sussman, MD, Department of Internal Medicine, NA10, Cleveland Clinic, 9500 Euclid Avenue, Cleveland, OH 44195; sussmat@ccf.org 